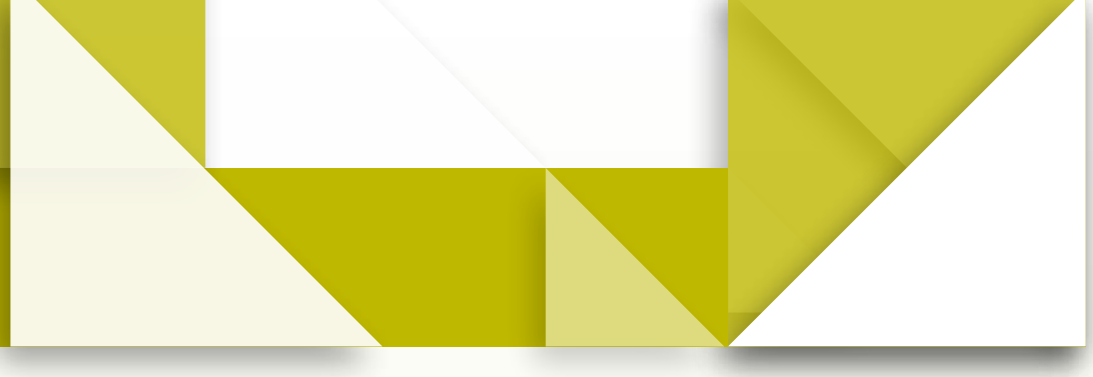

\title{
Laboratorio de química bajo contexto: insumo para el desarrollo de habilidades de pensamiento crítico
}

\section{- Chemical Laboratory on Context: Input for Development of Critical Thinking Skills \\ - Laboratório de química sob contexto: entrada para o desenvolvimento de habilidades de pensamento crítico}

\section{Resumen}

En la formación universitaria de profesionales afines a la química ha existido la preocupación por el desarrollo de la habilidad de pensamiento crítico; sin embargo, es claro que durante el desempeño como profesionales se identifican algunas dificultades en valerse de esta habilidad al tratar de resolver situaciones propias de su quehacer profesional. Una explicación probable a esta problemática es que, a pesar del esfuerzo de los docentes por diseñar nuevas estrategias de enseñanza, estas no han sido del todo efectivas, y por otro lado, se identifica que aún persiste en algunos profesores la concepción tradicional de enseñanza basada en un proceso memorístico. A fin de aportar al análisis de la problemática expuesta, este artículo de investigación, tipo mixta, muestra los resultados de un estudio en el que se aplican actividades y estrategias en torno a la química de los alimentos. Se trabajó con un grupo de estudiantes registrados en la asignatura de Química Agroalimentaria de la Licenciatura en Química de la Universidad Pedagógica Nacional, ubicada en Bogotá (Colombia), quienes adelantaron algunos experimentos bajo contextos determinados. Como resultado, se observa un favorecimiento en el desarrollo de habilidades de pensamiento crítico, evidenciado mediante la evaluación de una serie de mini proyectos.

Palabras clave

pensamiento crítico; enseñanza superior; resolución de problemas; enseñanza/aprendizaje; miniproyectos

\section{Rodrigo Rodríguez Cepeda* Jaime Augusto Casas Mateus**} Diana Esperanza Martínez Cárdenas* **

Doctor en Educación; profesor planta, Universidad Pedagógica Nacional.

rrodriguez@pedagogica.edu.co

* * Doctor en Educación; profesor planta, Universidad Pedagógica Nacional.

jcasas@pedagogica.edu.co

* * * Magíster en Docencia de la Química. Universidad Antonio Nariño. Docente Química Facultad de Ciencias. Coordinadora Pregrado en Bioquímica.

emartinez25@uan.edu.co

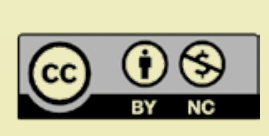

Primer semestre de 2020 / ISSN 2665-3184

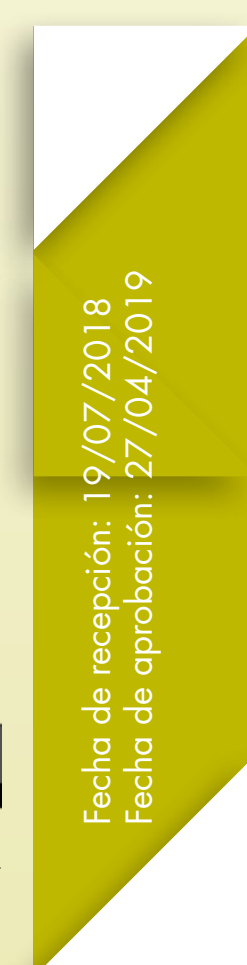




\section{Abstract}

In university education, of professionals related to chemistry, there has been a concern for the development of critical thinking skills. However, it is clear that during the performance as professionals, some difficulties are identified in using this skill when trying to solve situations own of his professional work. A probable explanation to this problem is that, despite the effort made by teachers to design new teaching strategies, these have not been entirely effective, and on the other hand, it is identified that still persists in some teachers the traditional conception of teaching based on a rote process. In order to contribute in the study of the exposed problems, this research shows the results of a study in which activities and strategies are applied around the chemistry of food. We worked with a group of students registered in the subject of Chemistry Agroalimentaria of the Degree Program in Chemistry of the National Pedagogical University, located in Bogotá - Colombia, who worked on some experiments under certain contexts. As a result, a favoring in the development of critical thinking skills is observed, evidenced by the evaluation of a series of mini-projects.

Keywords

critical thinking; didactic; teaching of science; problem solving; teaching learning; mini-projects

\section{Resumo}

No ensino universitário, dos profissionais ligados à química, tem havido uma preocupação com o desenvolvimento de habilidades de pensamento crítico. Porém, fica claro que durante a atuação como profissionais, algumas dificuldades são identificadas no uso dessa habilidade ao tentar resolver situações próprio de seu trabalho profissional. Uma provável explicação para este problema é que, apesar dos esforços feitos pelos professores para projetar novas estratégias de ensino, estes não têm sido totalmente eficazes, e em segundo lugar, identifica-se que a concepção tradicional de educação ainda persiste em alguns professores baseado em um processo de memorização. A fim de contribuir no estudo dos problemas expostos, esta pesquisa mostra os resultados de um estudo em que atividades e estratégias são aplicadas em torno da química da alimentação. Trabalhamos com um grupo de alunos matriculados na disciplina de Química Agroalimentar do Programa de Licenciatura em Química da Universidade Pedagógica Nacional, ubicada em Bogotá - Colômbia, quem trabalharam em algumas experiências em determinados contextos. Como resultado, observa-se um favorecimento no desenvolvimento de habilidades de pensamento crítico, evidenciado pela avaliação de uma série de mini projetos.

Palavras-chave

pensamento crítico; didático; ensino de ciências; resolução de problemas; ensino-aprendizagem; mini projetos 


\section{Introducción}

La importancia del aprender a aprender en los estudiantes es innegable, y en esta medida, mejorar la calidad del pensamiento se configura como uno de los grandes desafíos para los docentes en la actualidad. Así, la educación basada en competencias representa una estrategia válida para que los estudiantes desarrollen habilidades que les permitan enfrentar el mundo regido por la incertidumbre (Ortiz et ál., 2015).

En este sentido, la formación por competencias sugiere que la educación debe permitir la construcción de conocimientos como eje para el desarrollo del pensamiento; sin embargo, al parecer no garantiza el desarrollo de habilidades de pensamiento crítico, como lo señalan algunas investigaciones de los años 1980 (Whimbey, 1985; Perkins, 1985; Glaser, 1984).

Recientemente, algunos autores han mencionado la importancia de las propuestas curriculares tendientes a romper con la tendencia memorística en los procesos educativos, por cuanto estos parecen imposibilitar el desarrollo de habilidades del pensamiento crítico (Castillo y Martínez, 2017$)$. Por tanto, en la actualidad existe una abierta explosión de la información, donde la disponibilidad inmediata de ella, no necesariamente válida, requiere que las personas desarrollen el pensamiento crítico como una vía para seleccionar eficazmente la información pertinente; por lo que es importante reemplazar las tendencias memorísticas aún presentes en la educación (Santiago, 2016), por metodologías que lo incentiven.

De esta manera, es importante focalizar los esfuerzos de los docentes por estructurar y desarrollar propuestas que promuevan tales habilidades, desde acciones sistematizadas en los espacios académicos pertinentes. En este contexto, el presente artículo muestra los resultados de una intervención de aula, con estudiantes de noveno semestre del programa de Licenciatura en Química de la Universidad Pedagógica Nacional, registrados en el curso de énfasis en química agroalimentaria, con el fin de desarrollar las habilidades interpretativas y argumentativas del pensamiento crítico, en el marco de actividades contextualizadas a problemas relacionadas con la química de alimentos.

\section{Marco teórico}

\section{Habilidades de pensamiento crítico}

El aprendizaje centrado en la memoria ha sido ampliamente criticado, en especial cuando en la década de 1980 los investigadores abren la discusión alrededor del desarrollo de habilidades de pensamiento en el entorno escolar (Fancione, 1990), incluyendo los de tipo reflexivo, racional y crítico.

Algunos autores han tratado de definir pensamiento crítico: Robert Ennis y Millman (1985) lo entienden como un pensamiento racional y reflexivo centrado en por qué hacer o creer en un momento determinado. En otras palabras, privilegia la razón frente a otras dimensiones propias del pensamiento, en el que se vislumbra la tendencia a la acción, y del que surge la resolución de problemas como el contexto que condiciona el pensamiento crítico, para entender la naturaleza de los problemas más que en generar soluciones a estos.

Posteriormente, Ennis (2011) amplía la definición de pensamiento crítico en términos de habilidades (componente cognitivo) o de disposiciones (componente afectivo), de esta manera, se incluyen habilidades que le permiten al individuo emitir juicios confiables sobre la credibilidad de una afirmación o la conveniencia de una acción (Campos, 2007). Otros autores se refieren al pensamiento crítico como unas competencias metacognitivas y de evaluación epistemológica con implicaciones con el proceso de enseñanza (Saiz y Fernández, 2012; Kuhn y Weinstock, 2002).

Por otra parte, desde un marco psicológico, el concepto de habilidades de pensamiento crítico posee dos componentes: uno de orden cognitivo y otro más bien del dominio autorregulatorio, enmarcados en una habilidad de pensamiento complejo de alto nivel, 
incluyendo habilidades como comprensión, deducción, categorización y emisión de juicios valorativos, entre otras, todas ellas necesarias para lograr juicios críticos frente a una situación. En este sentido, Paul et ál. (1995) y Díaz-Barriga, (2001) concuerdan en que el pensamiento no es equivalente a la mera suma de habilidades específicas, separadas de sus contenidos y sus contextos propios.

Boisvert (2004) define el pensamiento crítico desde tres dimensiones: una estrategia de pensamiento, una investigación y un proceso; cuando se habla de estrategia de pensamiento, se hace referencia al conjunto de operaciones coordinadas necesarias para hacer cualquier actividad, ya sea tomar decisiones, resolver problemas o comprender un concepto o constructo.

Por otro lado, Kurfiss (1988) interpreta el pensamiento crítico como un proceso de investigación en el cual el cuestionamiento, la construcción de hipótesis y conclusiones que logran justificar diversos fenómenos, se presentan como aspectos importantes para que los individuos desarrollen las habilidades propias del pensamiento crítico.

Por último, varios autores lo conciben como un proceso activo donde actitudes apropiadas (amplitud de mente y honestidad intelectual) y una serie de capacidades de razonamiento y de investigación condicionan el desarrollo de las habilidades propias del pensamiento crítico (Zechmeiser y James, 1992). Por su parte, Brookfield, (1991) lo considera un proceso que consta de una alternancia entre una serie de fases de análisis y acción, fundamentales para tal proceso, dichas fases son:

1. La aparición de una situación que no se atendió.

2. Evaluación de la situación.

3. Búsqueda de explicaciones o de soluciones a la situación en estudio.

4. Concepción de diferentes perspectivas respecto a la situación.

5. Resolución de la situación.

Facione (2007) discrimina la interpretación, el análisis, la evaluación, la inferencia, la explicación y la autorregulación como las habilidades cognitivas esenciales para el desarrollo del pensamiento crítico. Igualmente, determina la curiosidad respecto a una amplia gama de asuntos, la preocupación por estar y mantenerse bien informado, y el estado de alerta frente a oportunidades para utilizar dicho pensamiento.

De la misma manera, considera que la confianza en los procesos de investigación razonados, la autoconfianza en las propias habilidades para razonar, la mente abierta respecto a visiones divergentes del mundo, la flexibilidad al considerar alternativas y opiniones de las opiniones de otras personas, son actitudes necesarias para que una persona, en realidad, cuente con un pensamiento crítico. En la tabla 1 se puede observar un resumen de cada habilidad propuestas por Facione (2007). 
Tabla 1. Habilidades cognitivas

\begin{tabular}{|c|c|c|}
\hline Habilidad & Intención & Subhabilidad \\
\hline Interpretación & $\begin{array}{l}\text { Comprender y expresar el significado o } \\
\text { la relevancia de una amplia variedad de } \\
\text { experiencias, situaciones, datos, eventos, juicios, } \\
\text { creencias, reglas, procedimientos o criterios. }\end{array}$ & $\begin{array}{l}\text { Categorización, decodificación del } \\
\text { significado y aclaración del sentido. }\end{array}$ \\
\hline Análisis & $\begin{array}{l}\text { Identificar las relaciones de inferencias reales } \\
\text { y supuestas entre enunciados, preguntas, } \\
\text { conceptos u otras formas de representación, } \\
\text { que tienen el propósito de expresar juicio, } \\
\text { experiencias, razones, información u opiniones. }\end{array}$ & $\begin{array}{l}\text { Examinar las ideas, detectar } \\
\text { y analizar argumentos. }\end{array}$ \\
\hline Evaluación & $\begin{array}{l}\text { Valorar la credibilidad de los enunciados } \\
\text { y de la fortaleza lógica de las relaciones } \\
\text { de inferencia, reales o supuestas, entre } \\
\text { enunciados, descripciones o preguntas. }\end{array}$ & \\
\hline Inferencia & $\begin{array}{l}\text { Identificar y asegurar los elementos necesarios } \\
\text { para sacar conclusiones razonables, formular } \\
\text { conjeturas e hipótesis y sacar las consecuencias } \\
\text { que se desprendan de los datos, enunciados, } \\
\text { principios, evidencia, juicios, opiniones, } \\
\text { conceptos, descripciones o preguntas. }\end{array}$ & $\begin{array}{l}\text { Cuestionar la evidencia, proponer } \\
\text { alternativas, y sacar conclusiones. }\end{array}$ \\
\hline Explicación & $\begin{array}{l}\text { Capacidad de presentar los resultados } \\
\text { del razonamiento propio de manera } \\
\text { reflexiva y coherente. }\end{array}$ & $\begin{array}{l}\text { Describir métodos y resultados, } \\
\text { justificar procedimientos. }\end{array}$ \\
\hline Autorregulación & $\begin{array}{l}\text { Monitoreo autoconsciente de las actividades } \\
\text { cognitivas propias, y de los resultados obtenidos, } \\
\text { aplicando particularmente habilidades de análisis } \\
\text { y de evaluación, con la idea de cuestionar, } \\
\text { confirmar o corregir el razonamiento propio. }\end{array}$ & El autoexamen y la autocorrección. \\
\hline
\end{tabular}

Fuente: tomado de Facione (2007).

\section{Instrumentos para evaluar el pensamiento crítico}

Una de las grandes dificultades para evaluar el pensamiento críico es el desarrollo de instrumentos que logren incorporar todas las habilidades propias de este. Sin embargo, hay una serie de instrumentos relacionados en documentos de la Association of American Colleges and Universities (Butler, 2012).

Sin embargo, han sido criticados en relación con su validez y su confiabilidad, con argumentos de corte conceptual, y otros de corte metodológico (Saiz y Rivas, 201 1). Las críticas obedecen, en unos casos, a la pluralidad de conceptos relacionados con el pensamiento crítico, mientras que en otros tantos, al tipo de formato de respuesta cerrada, ya que está en contravía de los principios del pensamiento crítico. nell Test of Critical Thinking, desarrollado por Ennis y Millman (1985) y aplicado por varios investigadores como Calle (2013). Este instrumento tiene la limitación de estar elaborado para jóvenes entre 9 y 18 años, con una estructura de opción múltiple, y evalúa las habilidades de inducción, credibilidad de una fuente, observación, semántica, deducción, y la identificación de hipótesis.

Pos su parte, el instrumento California Critical Thinking Skills Test (Facione, 1990) está diseñado para el contexto universitario y evalúa cinco habilidades cognitivas: interpretación, análisis, evaluación, explicación e inferencia. El constructo teórico del instrumento es compatible con la conceptualización del pensamiento crítico promulgado por el Sistema de la Universidad Estatal de California (Calle, 2013 ). 
En este orden de ideas, la presente investigación pretende validar, mediante juicios de expertos, un instrumento elaborado con preguntas abiertas, enfocadas a identificar las habilidades del pensamiento crítico de interpretar y argumentar, por parte de un grupo de estudiantes universitarios, registrados en los dos últimos semestres del programa de Licenciatura en Química. Por otra parte, se desarrollan actividades prácticas en el aula, enmarcadas en el concepto de miniproyectos contextualizados en los problemas propios de la química de alimentos.

\section{Justificación}

La química, por ser una ciencia experimental, requiere de personas con un pensamiento crítico desarrollado, con el fin de analizar, interpretar resultados, proponer hipótesis, inferir, evaluar y proponer explicaciones de fenómenos surgidos en las transformaciones de la materia. Por tanto, surge la necesidad de formar investigaciones en el ámbito educativo a nivel universitario, enfocadas al desarrollo explícito de habilidades de pensamiento crítico.

De esta manera, la presente investigación se justifica desde el campo del desarrollo de destrezas de interpretación, análisis e inferencia como habilidades del pensamiento crítico a partir del trabajo práctico en el laboratorio de química, sustentado en experimentos bajo el contexto de la química de alimentos.

Por otra parte, es importante diseñar un instrumento que identifique las habilidades del pensamiento crítico para una población con las características que esta investigación define, ya que los existentes son genéricos y no tienen en cuenta las particularidades de la población.

\section{Diseño metodológico}

El marco metodológico se inscribe en la investigación preexperimental, el cual incluye una serie de actividades en las que se involucra el diseño y puesta a punto de instrumentos para diagnosticar el grado de desarrollo de las habilidades de interpretar y argumentar en la población objeto de estudio. Posteriormente, se desarrollan trabajos experimentales en el laboratorio mediante miniproyectos, en los cuales se plantean problemas relacionados con la composición de alimentos, especialmente en los parámetros de humedad, grasa, carbohidratos, fibra y proteínas. Por último, se recurre al instrumento inicial para evidenciar el efecto de las actividades en la promoción de habilidades de pensamiento crítico.

El diseño metodológico consta de un conjunto de situaciones, actividades y experiencias a partir del cual se construye y reconstruye el conocimiento, y se ajusta al contexto sociocultural. Para el caso de esta investigación, se utilizan dos etapas: la inicial, en la que se diseña, valida y aplica el instrumento diagnóstico inicial; la segunda, que incluye el desarrollo de trabajos prácticos experimentales estructurados a manera de miniproyectos, monitoreados y evaluados desde la perspectiva de la promoción de habilidades del pensamiento crítico.

\section{Ełapa 1. Instrumento diagnóstico inicial}

Aquí se recolectan datos con el fin de identificar el nivel de desarrollo de las habilidades de pensamiento crítico en los estudiantes del programa de Licenciatura en Química, registrados en el ciclo de profundización (últimos tres semestres), 
teniendo en cuenta que uno de los objetivos del programa en este ciclo es desarrollar competencias investigativas.

Para ello se comienza con la aplicación de un instrumento inicial (anexo), previamente validado por expertos, que consta de seis preguntas abiertas que evalúan el grado de desarrollo de las habilidades de interpretación y argumentación, mediante situaciones problema en el contexto de la química de alimentos.
Etapa 2. Elaboración de material didáctico, trabajo experimental en el laboratorio de química y evaluación de habilidades de pensamiento crítico

Se plantea un trabajo mediante el uso de miniproyectos basados en la resolución de problemas, como estrategia didáctica. Con ellos se espera promover el desarrollo de las habilidades

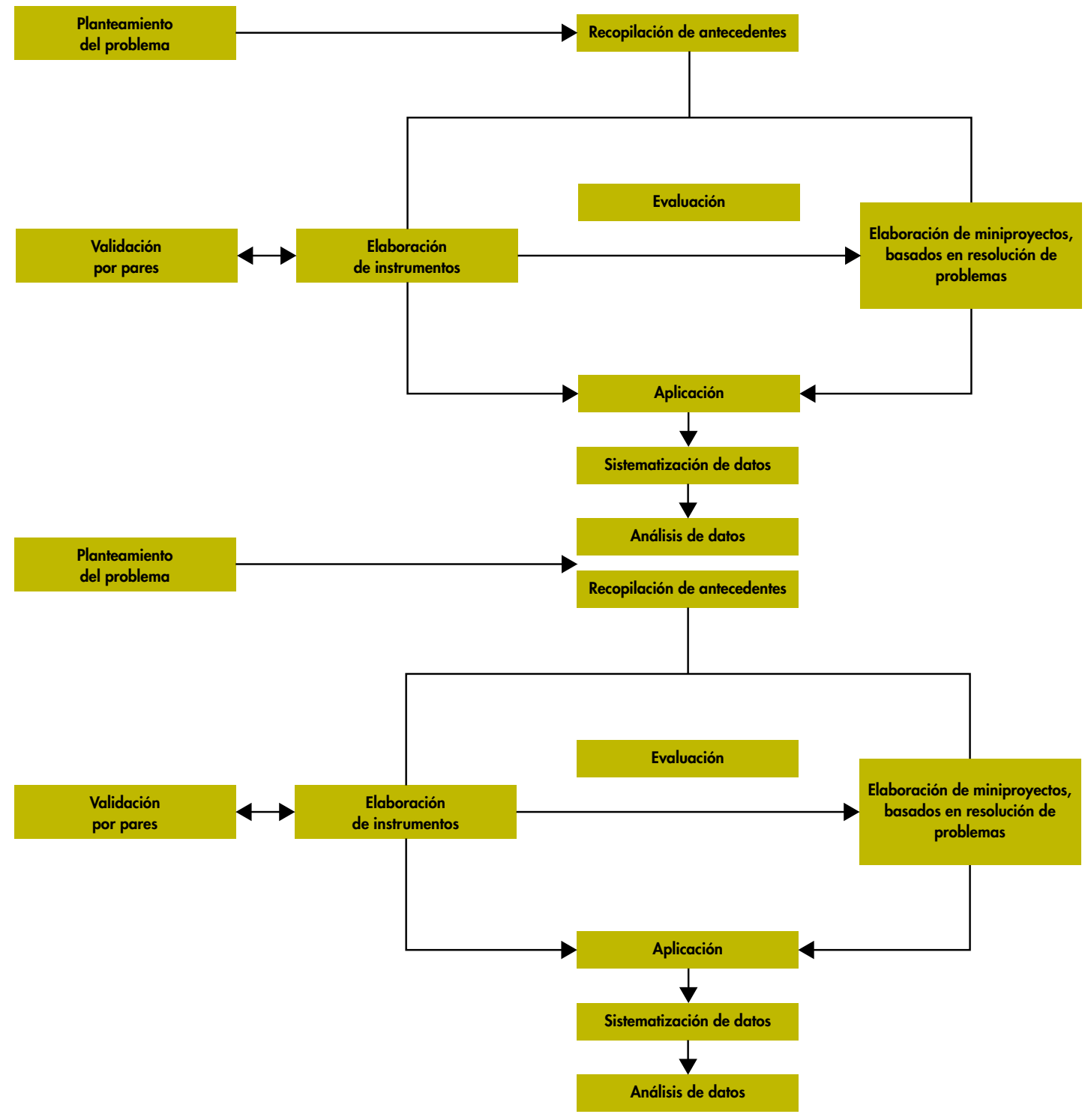

Figura 1. Metodología

Fuente: elaboración propia. 
interpretativas y argumentativas, propias del pensamiento crítico, mediante el planteamiento de situaciones problema en el contexto de la química de alimentos.

Los datos recolectados se sistematizan y analizan mediante el uso de los paquetes estadísticos SPSS y Atlas.ti, con el fin de identificar, estadísticamente, indicadores de correlación entre las actividades realizadas y el desarrollo de las habilidades; así como, identificar familias y redes de conceptos mediante el análisis de los textos presentados en los informes de laboratorio, de esta manera se espera identificar el grado de desarrollo de las habilidades del pensamiento crítico. En la figura 1 se resume la metodología utilizada.

\section{Población}

La población objeto de estudio estuvo constituido por 32 estudiantes del programa de Licenciatura en Química de la Universidad Pedagógica Nacional, inscritos en el ciclo de profundización, quienes han cursado por lo menos siete semestres académicos.

La presente investigación se fundamenta en los parámetros de un modelo preexperimental, bajo los postulados de una investigación mixta en la cual los datos se recolectan por series cronológicas con único grupo, sin grupo control, enfocado a analizar la influencia de la resolución de problemas en el desarrollo de algunas habilidades propias del pensamiento crítico planteado por Facione (2007).

\section{Resultados y discusión}

Durante la segunda mitad del siglo xx se hicieron reiterativos los cuestionamientos a las prácticas educativas basadas en la memoria, por lo que la sociedad empezó a percibir la necesidad de innovar los procesos educativos. Probablemente, la influencia de la revolución industrial hizo que las investigaciones en educación se enfocaran a promover actividades reflexivas (Santiago, 2016), en los cuales los estudiantes tienen un papel mucho más participativo en su formación.

En este contexto, se diseña un instrumento inicial con seis preguntas abiertas (anexo), el cual pretendía indagar sobre el nivel de desarrollo de las habilidades de argumentación e interpretación, utilizando la temática de proteínas en el contexto de los alimentos, este instrumento fue validado previamente por cinco expertos, todos ellos con estudios doctorales en educación, y experiencia en educación universitaria en química, uno experto en química de alimentos y otro con experiencia docente en química analítica, quienes valoraron cada pregunta mediante una matriz evaluativa (en la que se solicitaba marcar con una equis el valor que se considerara, teniendo en cuenta que 1 es muy bajo y 5 es muy alto) que se presenta en la tabla 2. 
Tabla 2. Matriz evaluativa para validación de instrumento

\begin{tabular}{|c|c|c|c|c|c|c|c|c|c|c|c|}
\hline & \multicolumn{4}{c|}{$\begin{array}{c}\text { Nivel de interpretación necesaria } \\
\text { para responder }\end{array}$} & \multicolumn{5}{c|}{$\begin{array}{c}\text { Nivel de argumentación } \\
\text { necesario para responder }\end{array}$} \\
\hline Pregunta & $\mathbf{1}$ & $\mathbf{2}$ & $\mathbf{3}$ & $\mathbf{4}$ & $\mathbf{5}$ & $\mathbf{1}$ & $\mathbf{2}$ & $\mathbf{3}$ & $\mathbf{4}$ & $\mathbf{5}$ \\
\hline 1 & & & & & & & & & & \\
\hline 2 & & & & & & & & & & \\
\hline 3 & & & & & & & & & & \\
\hline 4 & & & & & & & & & & \\
\hline 5 & & & & & & & & & & \\
\hline 6 & & & & & & & & & & \\
\hline
\end{tabular}

Nota: Marque con una equis $(\mathrm{X})$ el valor que considere teniendo en cuenta que 1 es muy bajo y 5 es muy alto.

Fuente: elaboración propia.

Se obtuvieron valores de alfa de Cronbach de 0,803 para interpretación y 0,702 para argumentación, lo cual indica una buena fiabilidad del instrumento para identificar estas habilidades del pensamiento crítico.

Una vez validado el instrumento inicial, se aplica al grupo de estudiantes y se evalúa con la matriz presentada en la tabla 2 . En la figura 2 se observa el resultado para la primera pregunta.

a. Las proteínas son aminoácidos, los cuales, por hidrólisis producen poliamidas unidas por un enlace característico que recibe el nombre de enlace peptídico, tal propiedad estructural permite medir la formación del amoniaco presente en una muestra alimenticia, debido al contenido de nitrógeno, llevando a cabo una reacción de oxidación, y determinando así la denominada proteína bruta.

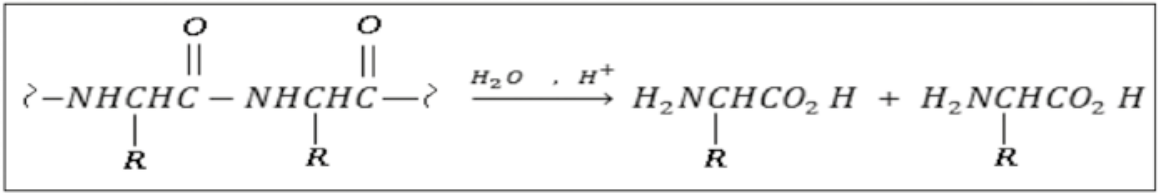

De acuerdo con la reacción anterior, ¿̇cree que existe alguna expresión que invalida el texto? Argumente su respuesta. 

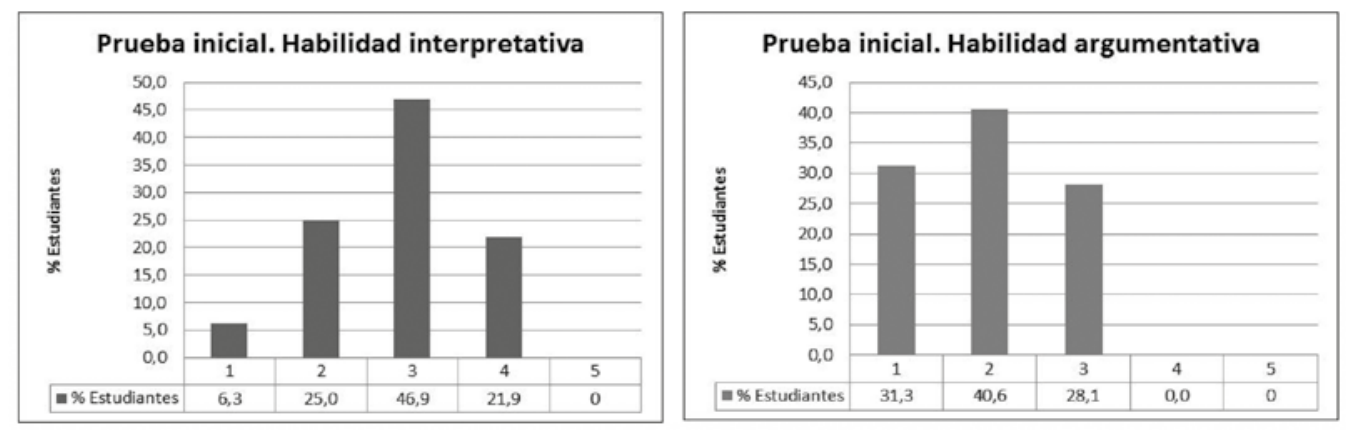

Figura 2. Resultados pregunta 1 pretest

Fuente: elaboración propia.

Las tendencias presentadas en el desarrollo de las dos habilidades estudiadas suponen que los estudiantes suelen interpretar el enunciado y proponen una respuesta a la problemática presentada, corroborado por casi el $69 \%$ de los estudiantes que obtienen una valoración entre 3 y 4 para esta habilidad (figura 2). Sin embargo, no cuentan con buenos argumentos para explicar la respuesta dada, ya que el $100 \%$ de los estudiantes son calificados entre 1 y 3 para esta habilidad.

La pregunta 2 pretende identificar el nivel de interpretación de la pregunta y la argumentación que realizan los estudiantes, utilizando conceptos químicos

b. Para la cuantificación de proteínas se realiza una oxidación con $\mathrm{H}_{2} \mathrm{SO}_{4}$ concentrado en presencia de un catalizador, el producto obtenido reacciona con un exceso de álcali fuerte para liberar $\mathrm{NH}_{3}$, siendo este último recogido en una solución de $\mathrm{H}_{3} \mathrm{BO}_{3}$ para formar el $\mathrm{NH}_{4} \mathrm{H}_{2} \mathrm{BO}_{3}$, finalmente, la anterior solución se titula con $\mathrm{HCl}$ estandarizado obteniéndose nuevamente el $\mathrm{H}_{3} \mathrm{BO}_{3}$. Formule las reacciones descritas. ¿ Será posible hallar solo el contenido de fenilalanina en una muestra de alimento? Indique el porqué de su respuesta.

En la figura 3 se observan los resultados.
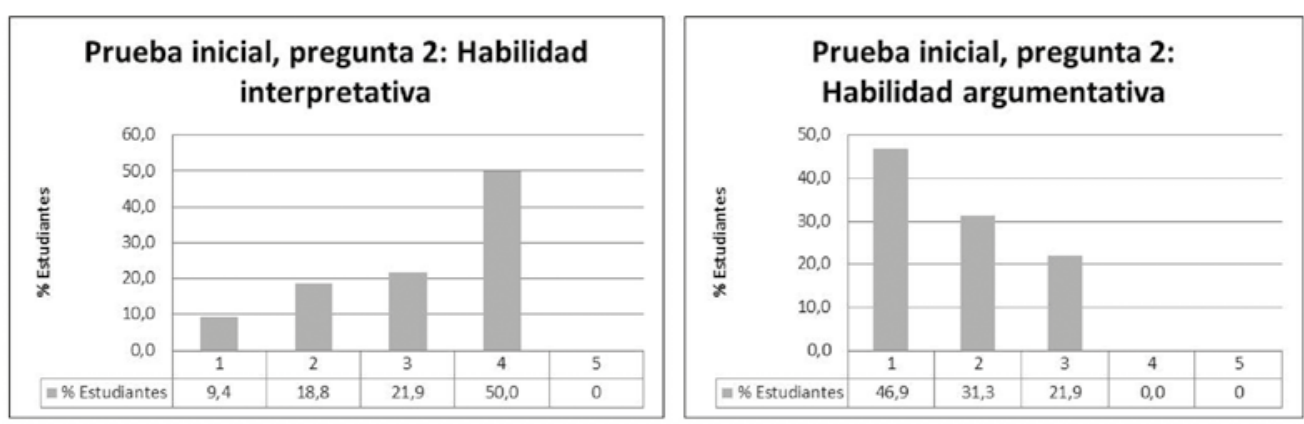

Figura 3. Resultados pregunta 2 pretest

Fuente: elaboración propia.

Teniendo en cuenta que a partir de una valoración igual o superior a 4 se asume como un buen nivel en la habilidad evaluada, estos resultados muestran 
que los estudiantes interpretan aceptablemente la pregunta, pero no pueden argumentar empleando conceptos químicos, a tal punto que, en muchos casos, ellos no responden la pregunta.

En términos generales, se observa la misma tendencia en las otras preguntas del pretest, donde las habilidades interpretativa y argumentativa muestran un bajo desarrollo. Se destaca que en general la habilidad interpretativa logra un mayor nivel. En la figura 4 se ilustra el resultado de la mediana aritmética del valor obtenido por cada habilidad en cada estudiante.

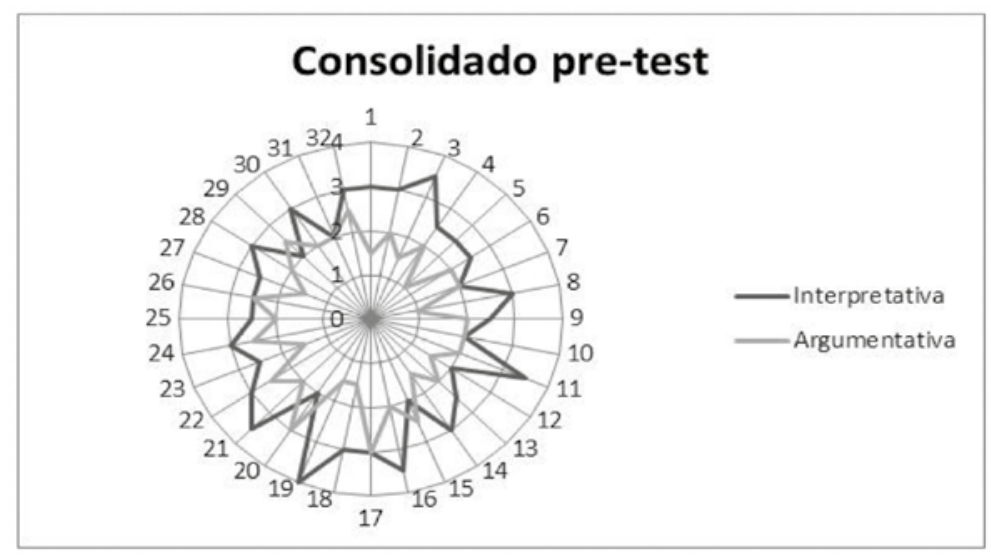

Figura 4. Mediana aritmética, valoración de la habilidad en cada estudiante

Fuente: elaboración propia.

Con estos resultados se elaboran actividades en el aula, mediante miniproyectos planteados por los estudiantes y ajustados según la orientación del profesor. Estos se sugieren en el contexto de la química de alimentos, específicamente en las temáticas del análisis proximal, lo cual facilita que cada grupo de estudiantes analice un alimento seleccionado por ellos, estimulando la formulación de actividades grupales desarrolladoras del pensamiento crítico, teniendo en cuenta que deben interpretar las actividades propuestas y sus referentes conceptuales, analizar las teorías y resultados en los trabajos prácticos de los miniproyectos, que los lleven a inferir conclusiones.

Esta metodología permite hacer el seguimiento de avance de los estudiantes, mediante una valoración cuantitativa y cualitativa, según los parámetros establecidos en la tabla 3, evaluados en los informes de cada miniproyecto sustentado por cada subgrupo:

Tabla 3. Valoración cuantitativa y cualitativa del desarrollo de los miniproyectos

\begin{tabular}{|c|c|}
\hline Intervalos & Calificativo \\
\hline 0 a 2,9 & Se debe realizar un mayor refuerzo para el desarrollo de las habilidades \\
\hline 3,0 a 3,9 & El estudiante demuestra un regular desarrollo de las habilidades propuestas \\
\hline 4,0 a 5,0 & El estudiante demuestra un buen desarrollo de las habilidades \\
\hline
\end{tabular}

Fuente: elaboración propia.

Los miniproyectos se diseñaron teniendo en cuenta la estructura presentada en la tabla 4, propuesta por Cárdenas (2001). 
Tabla 4. Estructura de los informes

\begin{tabular}{|c|l|}
\hline Secciones & \multicolumn{1}{|c|}{ Identificación } \\
\hline Sección A & Título \\
\hline Sección B & Nombres y fecha \\
\hline Sección C & Enunciado y condiciones \\
\hline Sección D & $\begin{array}{l}\text { Discusión del enunciado: mediante preguntas a resolver } \\
\text { donde el estudiante debe realizar una revisión teórica }\end{array}$ \\
\hline Sección E & $\begin{array}{l}\text { Interpretación del enunciado: espacio para que el estudiante diseñe un } \\
\text { trabajo práctico en el laboratorio a partir de la discusión del enunciado }\end{array}$ \\
\hline Sección F & $\begin{array}{l}\text { Materiales, reactivos y equipos: lista propuesta por los } \\
\text { estudiantes para desarrollar el trabajo práctico diseñado }\end{array}$ \\
\hline Sección G & $\begin{array}{l}\text { Reporte final: espacio diseñado para que el estudiante analice e infiera } \\
\text { sobre los resultados obtenidos en los trabajos prácticos de laboratorio }\end{array}$ \\
\hline
\end{tabular}

Fuente: elaboración propia.

\section{Miniproyecto proteínas}

Para el desarrollo de este miniproyecto se plantea una pregunta orientadora, cuya intención es motivar a los estudiantes para plantear una metodología de análisis tendiente a cuantificar proteína en un alimento; igualmente, se proponen diversas preguntas alrededor del tema, en especial frente a la dicotomía aminoácido y proteína, la química de los aminoácidos y su relación con la nutrición. Por último, el miniproyecto pide al estudiante interpretar la pregunta orientadora y analizar los resultados después de desarrollada la actividad práctica.

Como se ilustra en la figura 5, los estudiantes tienen dificultades en la habilidad de interpretación ya que no describen con facilidad la situación problema ni los resultados, lo que requiere un gran apoyo por parte del profesor.

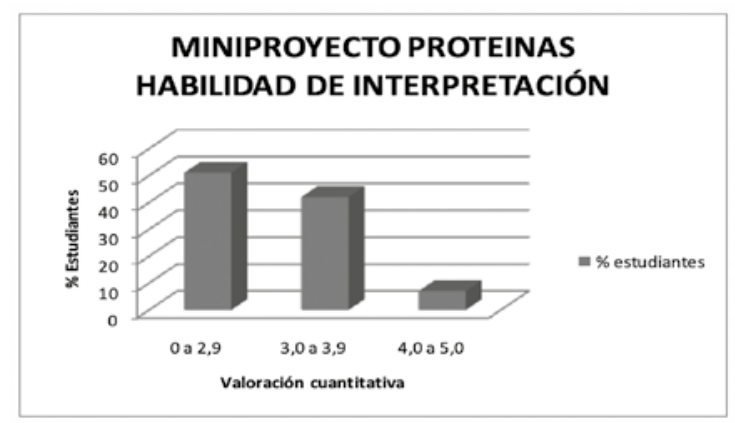

Figura 5. Desarrollo de habilidades interpretativas en el miniproyecto de proteínas

Fuente: elaboración propia.

De acuerdo con los resultados anteriores, es indispensable que el profesor oriente a los estudiantes en la interpretación del problema y en el análisis de los resultados, para lo cual es necesario realizar lecturas de informes de laboratorio y artículos. Esto posibilita el aumento del nivel de lectura científica con conceptos asociados a las proteínas; por otro lado, los estudiantes presentan los resultados en un informe de laboratorio, del que se percibe un análisis crítico. 


\section{Miniproyecto grasas}

Este miniproyecto cuenta con la misma estructura planteada, y las preguntas se centran en las propiedades de los solventes para extraer la fracción lipídica de un alimento, así como en los diferentes métodos analíticos para extraer y cuantificar grasas en alimentos.

Una vez evaluados los informes, se observa un mejor resultado en las habilidades del pensamiento crítico; los estudiantes hacen una mejor interpretación de los resultados, emplean lenguaje científico y apoyan sus análisis con diversas fuentes bibliográficas, lo que evidencia la lectura de artículos y libros (figura 6).

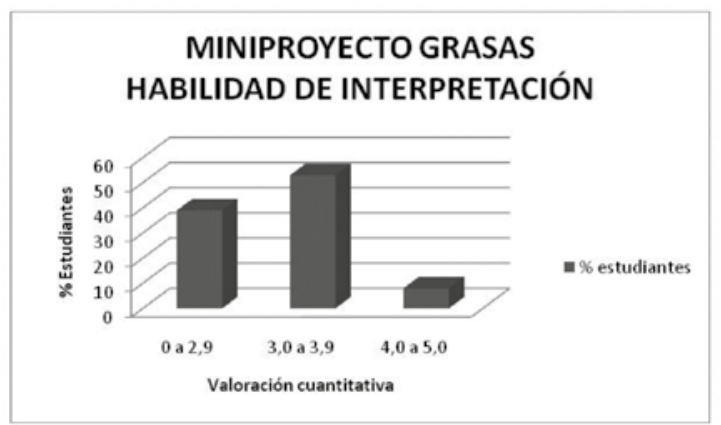

Figura 6. Desarrollo de habilidades

interpretativas, miniproyecto de grasas

Fuente: elaboración propia.

En este sentido, los trabajos de laboratorio realizados mediante miniproyectos permiten que se desarrollen algunas capacidades propias del pensamiento crítico. Pawluk (2012) menciona que los alumnos con quienes se trabaja esta metodología cuentan con mayor tendencia hacia actitudes favorables, iniciativa, creatividad, autonomía, espíritu crítico y trabajo participativo, lo que los convierte en sujetos activos en su proceso de formación.

De la misma manera, la aplicación de los miniproyectos de carbohidratos y fibra indica un desarrollo del interés por realizar lecturas de material científico, como artículos y libros de consulta; así como un mejoramiento en las habilidades de pensamiento crítico, probablemente por la facilidad de integrar lo teórico con lo experimental y lo metodológico en el laboratorio, al intentar resolver preguntas (Flores, Caballero y Moreira, 2009).

Una vez desarrollados y evaluados todos los trabajos prácticos, se observa la evolución de los estudiantes frente al desarrollo de algunas habilidades del pensamiento crítico; de esta manera, en el primer miniproyecto, el $60 \%$ de los estudiantes tenía una evolución regular de las habilidades del pensamiento crítico. Sin embargo, a medida que se avanza en la aplicación de los trabajos prácticos, la tendencia cambia, hasta alcanzar un $70 \%$ de los estudiantes con un buen desarrollo de las habilidades (figura 7).

El resultado anterior presume que, mediante la utilización de los trabajos de laboratorio, fundamentados en experimentos bajo contextos específicos, con preguntas orientadoras, es posible fomentar o desarrollar las habilidades que integran el pensamiento crítico, especialmente las habilidades de interpretar, analizar e inferir. Este aspecto es importante, si se tiene en cuenta que algunos investigadores resaltan las dificultades de fomentar el pensamiento crítico en escenarios educativos, a pesar de reconocer su importancia en los procesos académicos (Solbes y Torres, 2013 ).

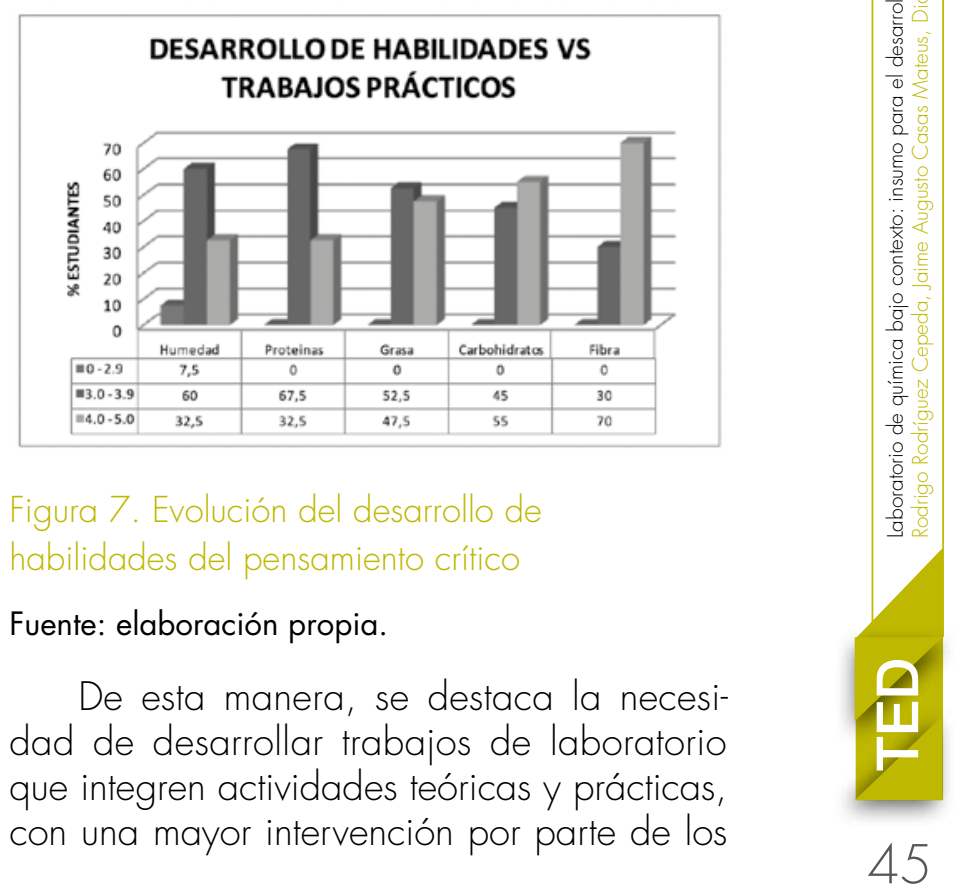


estudiantes en la planeación, diseño y desarrollo del laboratorio, basados en preguntas orientadoras a resolver, aspecto denominado laboratorios de tercer nivel, que se fundamentan en la fenomenología, y se centran en la comprensión y el aprendizaje (Sandi-Urena y Chrzanowski, 2016).

La aplicación del postest muestra que los estudiantes mejoraron la habilidad interpretativa, pero se observa un mejor desarrollo de la argumentativa. Como indica la figura 8, en esta tendencia, los trabajos de laboratorio en contexto, donde el estudiante se involucra en su diseño y aplicación, permiten adquirir lenguaje científico, el cual posibilita en ellos el análisis de los resultados y argumentar las conclusiones e hipótesis que se plantean.

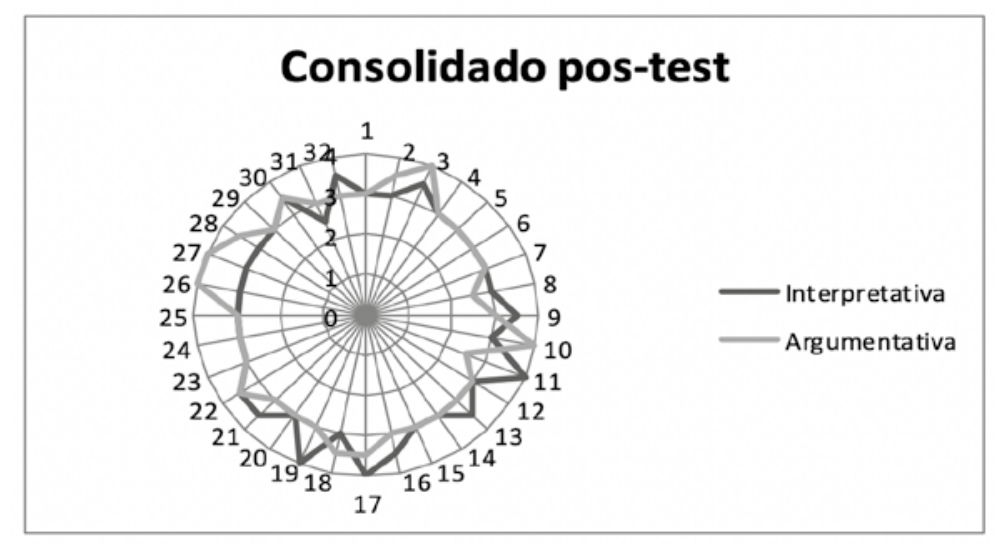

Figura 8. Media aritmética, valoración de las habilidades en cada estudiante

Fuente: elaboración propia.

En este sentido, emerge la necesidad de diseñar material didáctico que apoye el desarrollo de la indagación en los estudiantes, como un quehacer práctico en el laboratorio, evitando la descontextualización y la desarticulación entre lo teórico y lo experimental (Toma y Greca, 2015).

Por otro lado, al utilizar el paquete estadístico SPSS y comparar las medias aritméticas de las habilidades trabajadas en el pretest y postest, se encuentra una fuerte correlación entre la valoración de la habilidad interpretativa. En la tabla 5 se detallan los resultados para los estadísticos $\tau$ b de Kendall y $\rho$ de Spearman.

Tabla 5. Correlación bivariada habilidad interpretativa

\begin{tabular}{|c|c|c|c|}
\hline Estadísticos & Variables & & $\begin{array}{l}\text { Habilidad interpretativa } \\
\text { pos test }\end{array}$ \\
\hline \multirow{3}{*}{$\tau$ b de Kendall } & \multirow{3}{*}{$\begin{array}{c}\text { Habilidad } \\
\text { interpretativa pretest }\end{array}$} & Coeficiente de correlación & $0,583 * *$ \\
\hline & & Significancia (bilateral) & 0,001 \\
\hline & & Número de datos (N) & 32 \\
\hline \multirow{3}{*}{$\rho$ de Spearman } & \multirow{3}{*}{$\begin{array}{c}\text { Habilidad } \\
\text { interpretativa pretest }\end{array}$} & Coeficiente de correlación & $0,608 * *$ \\
\hline & & Significancia (bilateral) & 0,000 \\
\hline & & Número de datos (N) & 32 \\
\hline
\end{tabular}

* * La correlación es significativa en el nivel 0,01

Fuente: elaboración propia. 
Como se observa en la tabla 5, según los coeficientes estadísticos, existe una fuerte correlación entre el estado inicial de la habilidad interpretativa, evaluada en el pretest, con el resultado del postest. Este resultado puede indicar que los estudiantes evolucionan en su habilidad interpretativa sin desconocer sus habilidades previas a la intervención.

En la figura 9 se ilustra una red de conceptos, obtenida mediante análisis documental de un informe final, empleando el software Atlas-ti.

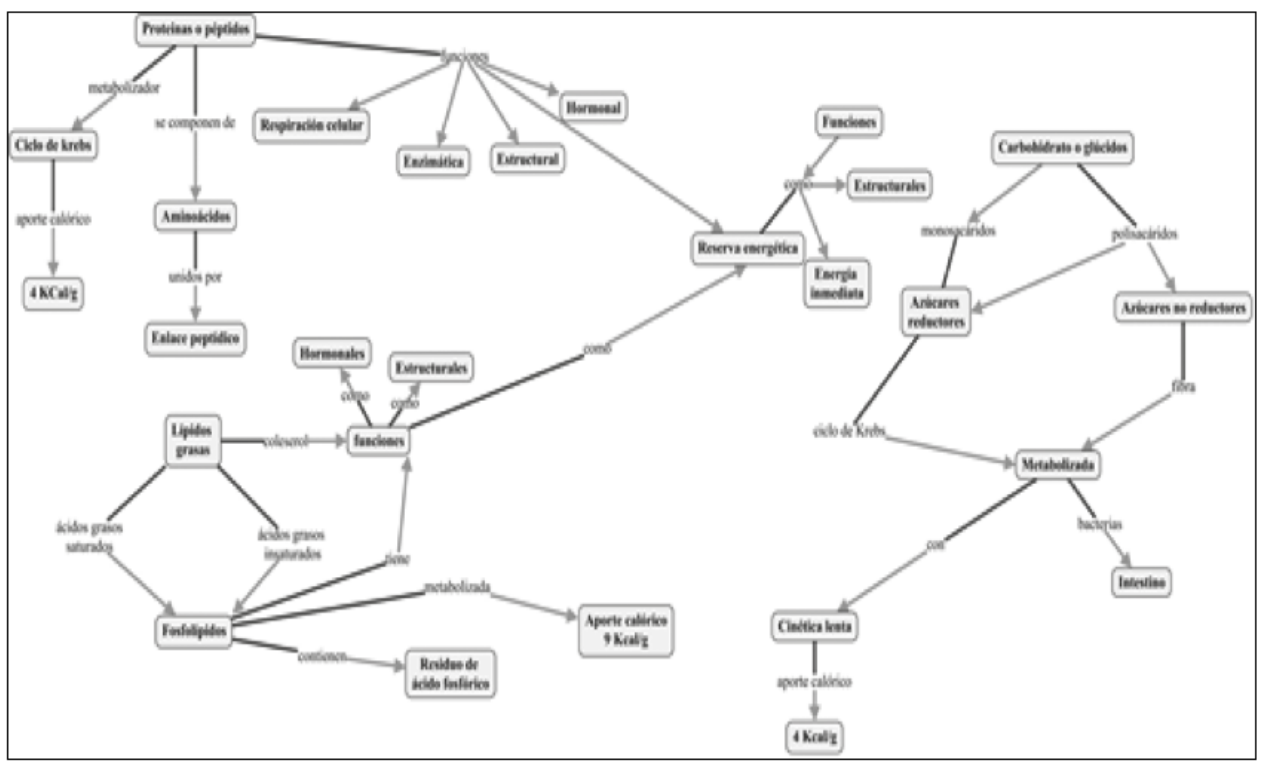

Figura 9. Red de códigos informe de laboratorio

Fuente: elaboración propia.

Como se observa, la red es compleja, debido al alto número de relaciones entre diversos conceptos. Sin embargo, también hay una alta coherencia, lo cual puede ser un indicativo de la evolución en la habilidad interpretativa, teniendo en cuenta que los estudiantes debían realizar un análisis de resultados. De esta manera, se puede concluir que los trabajos prácticos favorecen la evolución de la habilidad interpretativa en los estudiantes, así como la construcción de conceptos, como lo menciona Rodríguez-Cepeda (2016).

Por otra parte, si bien se observó una mejoría en la habilidad argumentativa, al realizar un análisis estadístico, no se encuentra una correlación definida ltabla 6): esta tendencia podría significar que los argumentos iniciales no inciden en la evolución o el desarrollo de esta habilidad, pero que los trabajos prácticos enmarcados en miniproyectos contextualizados facilitan el desarrollo de esta habilidad.

Tabla 6. Correlación bivariada. Habilidad argumentativa

\begin{tabular}{|c|c|c|c|}
\hline Estadísticos & Variables & $\begin{array}{c}\text { Habilidad argumentativa } \\
\text { pos test }\end{array}$ \\
\hline \multirow{2}{*}{$\tau$ b de Kendall } & $\begin{array}{c}\text { Habilidad } \\
\text { argumentativa } \\
\text { pretest }\end{array}$ & Coeficiente de correlación & 0,132 \\
\cline { 2 - 4 } & Significancia (bilaterall & 0,453 \\
\cline { 2 - 4 } & Número de datos $(\mathrm{N})$ & 32 \\
\hline
\end{tabular}




\begin{tabular}{|c|c|c|c|}
\hline \multirow{2}{*}{ Estadísticos } & Variables & $\begin{array}{c}\text { Habilidad argumentativa } \\
\text { pos test }\end{array}$ \\
\hline \multirow{2}{*}{$\rho$ de Spearman } & $\begin{array}{c}\text { Habilidad } \\
\text { argumentativa } \\
\text { pretest }\end{array}$ & Coeficiente de correlación & 0,135 \\
\cline { 2 - 3 } & Significancia (bilateral) & 0,462 \\
\hline
\end{tabular}

Fuente: elaboración propia.

\section{Conclusiones}

Teniendo en cuenta que en el pensamiento crítico convergen muchas habilidades -inferencia, interpretación, análisis, entre otras-, es importante que los programas de formación profesional las desarrollen en sus estudiantes, y más en las escuelas de formación de profesores, ya que sus egresados son los llamados a estructurar competencias en la escuela primaria, básica y media vocacional.

Como lo muestra esta investigación, la población objeto de estudio presentaba bajos niveles de desarrollo en las habilidades interpretativa y argumentativa, aspecto que podría ser consecuencia del uso de estrategias didácticas desarticuladas con los temas de interés para los estudiantes. En consecuencia, el diseño de actividades en el aula, contextualizadas y de interés para los estudiantes, parecen tener gran incidencia en el grado de desarrollo de las habilidades propias del pensamiento crítico.

Según los resultados, es factible suponer que los trabajos prácticos contextualizados, articulados con ejercicios de lectura de las temáticas, junto con el empleo de miniproyectos, favorecen la promoción de las habilidades argumentativas e interpretativas, por lo que estas actividades, deberían ser tenidas en cuenta en el diseño de programas de formación de profesores.

Se presume que los trabajos prácticos, enmarcados en miniproyectos, favorecen el desarrollo de la habilidad interpretativa, pero es importante tener en cuenta que su evolución presenta una fuerte correlación con el estado inicial de dicha habilidad. Bajo este panorama, se asume que las actividades en el aula, basadas en una evaluación inicial de las habilidades de pensamiento crítico, facilitarán la orientación de la educación desde otra perspectiva.

\section{Agradecimientos}

Los autores de este trabajo expresan sus agradecimientos al Centro de Investigaciones de la Universidad Pedagógica Nacional (CIUP), por la financiación del proyecto. Al Departamento de Química de la UpN, por permitir el uso de la planta física para realizar los trabajos prácticos. Al grupo de investigación Didáctica y sus Ciencias. Finalmente, a todos los estudiantes del Departamento de Química del énfasis en Química Agroalimentaria que participaron en la aplicación de este trabajo de investigación. 


\section{Referencias}

Boisvert, J. (2004). La formación del pensamiento crítico. Teoría y práctica. la. ed. México: Fondo de Cultura Económica.

Brookfield, S. (1991). Developing critical thinkers: Challenging adults to explore alternative ways of thinking and acting. Londres: Wiley.

Butler, H. (2012). Halpern critical thinking assessment predicts real-world outcomof critical thinkinges. Applied Cognitive Psychology, 26, 721-729.

Calle, G. (2013). La evaluación de las habilidades del pensamiento crítico asociadas a la escritura digital. Revista Virtual Universidad Católica del Norte, 40, 68-83.

Campos, A. (2007). Pensamiento crítico. Técnicas para su desarrollo. la. ed. Bogotá, Colombia: Cooperativa Editorial Magisterio.

Cárdenas, F. (2001). Miniprojects: An alternative to improve general Chemistry Teaching in higer education. Journal of Science Education, 2(2), 102-105.

Castillo, J. y Martínez, L. (2017). Caracterización de capacidades de pensamiento crítico en futuros profesores de química. Tecné, Episteme y Didaxis: TED, (número extraordinario), 504-5 11 . http://revistas. pedagogica.edu. co/index.php/TED/article/view/4567.

Díaz-Barriga, F. (200 1). Habilidades de pensamiento crítico sobre contenidos históricos en alumnos de bachillerato. Revista Mexicana de Investigación Educativa, 6(13), 1-19.

Ennis, R. (2011). The nature of critical thinking: an outline of critical thinking dispositions and abilities. Cambridge: Mrt. https://education. illinois.edu/docs/default-source/faculty-documents/robert-ennis/thenatureofcriticalthinking_51711_000.pdf

Ennis, R. y Millman, J. (1985). Cornell critical thinking test level. Pacific Grove C.A.: Midwest Publication.

Facione, P. (1990). Critical Thinking: A Statement of Expert Consensus for Purposes of Educa- tional Assessment and Instruction. ERIC Doc., ED 315 423, 1-3. San José: The Complete American Philosophical Association Delphi Report, The California Academic Press.

Facione, P. (2007). Pensamiento crítico: ¿Qué es y por qué es importante? Insight Assestment. https://www. insightassessment.com/About-Us/Measured-Reasons/pdf-file/Pensamiento-CriticoQue-es-y-por-que-es-importante/(language)/ esl-ES

Flores, J., Caballero, M. y Moreira, M. (2009). El laboratorio en la enseñanza de las ciencias: una visión integral en este complejo ambiente de aprendizaje. Revista de Investigación, 68, 75-111.

Glaser, R. (1984). The role of knowledge. American Pshycologist, 39, 93-104.

Kuhn, D. y Weinstock, M. (2002). What is epistemological thinking and why does it matter? En B. Hofer y P. Pintrich (eds.), Personal epistemology: The psychology of beliefs about knowledge and knowing (pp. 121-144). Nueva Jersey: Lawrence Erlbaum Associates Publishers.

Kurfiss, J. (1988). Critical Thinking: Theory, research, practice and possibilities. ASHEEric Higer Education Reports. Washington, D.C.: Association for the Study of Higher Education, ERIC Clearinghouse on Higher Education.

Ortiz, J., Fonseca, G., Cantín, M., Huitzil, E. y Lucena, J. (2015). Competencias genéricas para la educación en odontología forense: pensamiento crítico y responsabilidad social. International Journal of Odontostomatology, 9(2), 263-272.

Paul, R., Brinker, A., Martín, D., Veterano, C. y Kreklau, H. (1995). Critical Thinking Handbook: 6th and 9th grades. A guide for remodeling lesson plans in languaje arts. Social Students \& Science. Santa Rosa: Foundation for Critical Thinking.

Pawluk, D. (2012). Implementación de miniproyectos de investigación: una forma no con- 
vencional en los trabajos prácticos de práctica profesional de bioquímica. Santa Fe, Argentina: Universidad Nacional del Litoral.

Perkins, D. (1985). Postprimary education has a little inpact on informal reasoning. Journal Educational Pshycology, 77, 562-570.

Rodríguez-Cepeda, R. (2016). Aprendizaje de conceptos químicos: una visión desde los trabajos prácticos y los estilos de aprendizaje. Revista de Investigación, Desarrollo e Innovación, 7(1), 63-76.

Saiz, C. y Fernández, S. (2012). Pensamiento crítico y aprendizaje basado en problemas cotidianos. Revista de Docencia Universitaria, 10(3), 325-346.

Saiz, C. y Rivas, S. (201 1). Evaluation of the ARDEOS program: An initiative to improve critical thinking skills. Journal of the Scholarship of Teaching and Learning, 11(2), 34-51.

Sandi-Urena, S. y Chrzanowski, M. (2016). Learning in the Tertiary Level Chemistry Laboratory: What We Have Learnt from Phenomenology Research. Science Education Research and Practical Work, 181-192.

Santiago, J. (2016). La acción didáctica de las ciencias sociales y el desarrollo del pensamiento crítico. Revista Educación y Humanismo, 18(31), 241-256.

Solbes, J. y Torres, N. (2013). ¿Cuáles son las concepciones de los docentes de ciencias en formación y en ejercicio sobre el pensamiento crítico? Revista Tecné, Epistemé y Didaxis: TED, 33, 61-85.

Toma, R. y Greca, I. (2015). Enseñanza de las ciencias naturales a través de la metodología de indagación: un estudio de las unidades didácticas elaboradas por el alumnado del grado en Maestro de Educación Primaria. En M.C. Caballero, J.A. Meneses y M.A. Moreira (coords.), Actas del V Encuentro Iberoamericano sobre Investigación en Enseñanza de las Ciencias (pp. 479-490). Burgos: Universidad de Burgos.

Whimbey, A. (1985). You don't need a special reasoning test to implement and evaluated reasoning training. Educational Leadership, 43(2), 37-39.

Zechmeiser, E. y James, J. (1992). Critical Thinking. A functional approach. California: Brooks/Cole Publishing Company Pacific Grove.

\section{Para citar este artículo}

Rodríguez-Cepeda, R., Casas-Mateus, J. y Martínez-Cárdenas, D. (2020). Laboratorio de química bajo contexto: insumo para el desarrollo de habilidades de pensamiento crítico. Tecné, Episteme y Didaxis: TED, (47), 33-52. https://doi. org/10.17227/ted.num47-1 1334 


\section{Anexo}

\section{Universidad Pedagógica Nacional \\ Facultad de Ciencia y Tecnología \\ Departamento de Química \\ Prueba diagnóstica sobre proteínas}

1. Lea el siguiente escrito:

Las proteínas son aminoácidos, los cuales por hidrólisis producen poliamidas, unidas por un enlace característico que recibe el nombre de enlace peptídico. Tal propiedad permite medir la formación del amoniaco al hacer reaccionar la proteína con ácido y posteriormente con una base fuerte, de esta manera, es posible cuantificar la proteína presente en una muestra alimenticia y evaluar su aporte calórico.
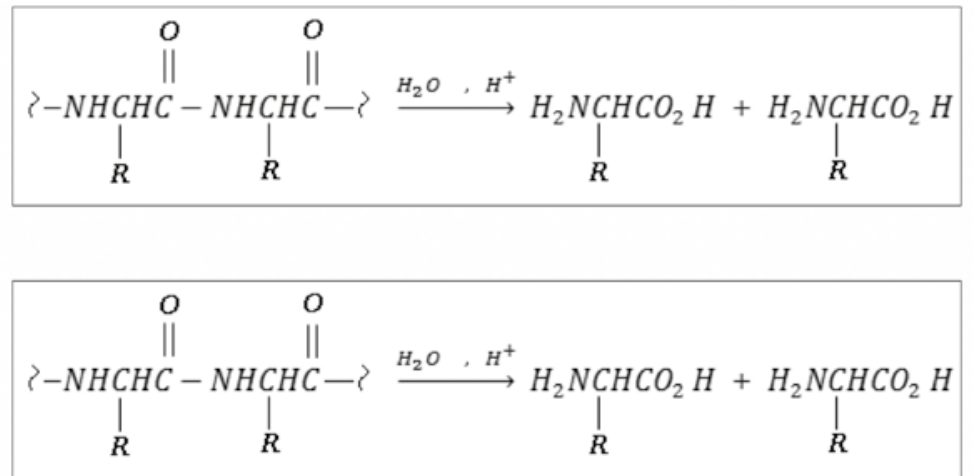

De acuerdo con el esquema anterior, ¿̇cree que existe algún argumento inválido en el texto? Enúncielo.

2. La estructura peptídica de las proteínas se puede diferenciar de otros compuestos orgánicos mediante varias pruebas químicas, por ejemplo, la hidrólisis de proteínas con ácidos, bases o enzimas para generar péptidos y finalmente aminoácidos.

¿Será posible hallar el contenido de fenilalanina en una muestra de alimento, si se oxida tal muestra con $\mathrm{H}_{2} \mathrm{SO}_{4}$ concentrado en presencia de un catalizador, el producto obtenido reacciona con un exceso de álcali fuerte para liberar amoniaco, el cual reacciona con una solución de $\mathrm{H}_{3} \mathrm{BO}_{3}$, para formar el $\mathrm{NH}_{4} \mathrm{H}_{2} \mathrm{BO}_{3}$, y finalmente, titular con $\mathrm{HCl}$ estandarizado? Indique el porqué de su respuesta y formule las ecuaciones de las reacciones descritas.

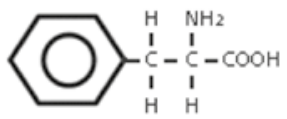

Fenilalanina

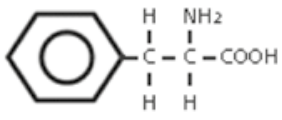


3. El anterior método analítico se basa en la medición del amoniaco formado por todo el nitrógeno presente en una muestra; en consecuencia, ¿̇sería posible afirmar que el porcentaje de nitrógeno total obtenido se debe solo al contenido de proteína presente en un alimento? ¿Por qué?

4. Un estudiante de química agroalimentaria inicia una serie de prácticas experimentales por triplicado para determinar el contenido de proteína en una muestra de pimentón, obteniendo los siguientes valores experimentales en la titulación final del proceso con $\mathrm{HCl}(0,100 \mathrm{~N})$ :

\begin{tabular}{|c|c|c|}
\hline Muestra & Masa muestra $(\mathbf{g})$ & Volumen $(\mathbf{m L})$ \\
\hline Blanco & - & 0,1 \\
\hline 1 & 7,62 & 1,1 \\
\hline 2 & 7,60 & 1,1 \\
\hline 3 & 7,65 & 1,2 \\
\hline
\end{tabular}

$\% N=\frac{V(m L) * N * \frac{14}{1000}}{W(g)} * 100$ además de que: \% Protev? na $=\% N * 6,25$

Con $V=$ ácido clorhídrico usado en la titulación (ml) $N=$ normalidad del ácido estándar $W(g)$ peso en gramos de la muestra

Con el fin de determinar el porcentaje de proteína presente en la muestra, un estudiante aplicó las anteriores fórmulas y determinó que en $100 \mathrm{~g}$ de esta muestra hay $0,02 \mathrm{~g}$ de nitrógeno total y $0,15 \mathrm{~g}$ de proteína. De acuerdo con esta información, ¿̇cuáles son las unidades químicas utilizadas en este tratamiento matemático?

5. Un analista químico encuentra que el contenido de nitrógeno total en una muestra de trigo y habichuela es el mismo, pero al determinar el nitrógeno proteico encuentra que debe utilizar factores de acuerdo con la siguiente tabla:

\begin{tabular}{|c|c|}
\hline Alimentos & Factor \\
\hline Carne, pescado, huevo, leguminosas & 6,25 \\
\hline Cereales y derivados de soya & 5,7 \\
\hline Leche & 6,38 \\
\hline Gelatina & 5,55 \\
\hline Arroz & 5,95 \\
\hline
\end{tabular}

¿Cuál es la razón por la que se debe usar factores diferentes en este tipo de análisis de alimentos?

6. Estudiantes de Química Agroalimentaria encuentran un método para determinar el contenido de proteína en una muestra alimenticia, en la primera fase se les pide realizar una digestión con $\mathrm{H}_{2} \mathrm{SO}_{4}$ concentrado, una mezcla catalizadora y la muestra a analizar indicándose temperaturas entre $370-410^{\circ} \mathrm{C}$, esto para que la pérdida de amoniaco no sea alta durante la digestión. ¿¿Qué función cumple el ácido sulfúrico y la mezcla catalizadora a la temperatura utilizada? 